\title{
Use of MALDI-TOF mass spectrometry in a 51- mutation test for cystic fibrosis: Evidence that 3199del6 is a disease-causing mutation
}

Inge M. Buyse, PhD ${ }^{1}$, Sarah E. McCarthy ${ }^{1}$, Paul Lurix, PhD ${ }^{1}$, Robert P. Pace, MS $^{1}$, David Vo ${ }^{1}$, George A. Bartlett ${ }^{1}$, Eric S. Schmitt, PhD, MS $S^{1}$, Patricia A. Ward, $M S^{1}$, Christopher Oermann, $M D^{2}$, Christine M. Eng, MD ${ }^{1}$, and Benjamin B. Roa, $P h D^{1}$

\begin{abstract}
Purpose: We developed a 51-mutation extended cystic fibrosis (CF) panel that incorporates the 25 previously recommended CFTR mutations, plus 26 additional mutations including 3199del6, which was associated with 1148T. Methods: This assay utilizes an integrated matrix-assisted laser desorption ionization-time of flight (MALDI-TOF) mass spectrometry system. Results: CF testing was performed on over 5,000 individuals, including a 3-year-old Hispanic-American patient with a compound heterozygous G542X/3199del6 genotype. He is negative for I148T, or other mutations assessed by CFTR gene sequencing. Conclusion: These results demonstrate the successful implementation of MALDI-TOF mass spectrometry in CF clinical testing, and establish 3199del6 as a disease-causing CF mutation. Genet Med 2004:6(5):426-430.
\end{abstract}

Key Words: cystic fibrosis, molecular testing, CFTR mutation, MALDI-TOF Mass spectrometry

Cystic fibrosis $(\mathrm{CF})$ is the most common life-threatening autosomal recessive disorder in the Caucasian population. The disease-causing CFTR gene lies on chromosome 7q31.2 and was cloned in $1989 . .^{1-3}$ The clinical spectrum ranges from a severe CF phenotype characterized by progressive lung disease, pancreatic insufficiency, male infertility, and elevated sweat chloride, to a milder presentation that includes congenital bilateral absence of the vas deferens (CBAVD) in males. ${ }^{4}$ Over 1300 CFTR mutations have been identified to date, wherein certain mutations are correlated with a mild or severe clinical phenotype. ${ }^{5} \mathrm{CF}$ carrier screening for couples of reproductive age is recommended in the U.S. using a core panel of 25 common CF mutations. ${ }^{6}$ Although laboratories vary in the number of mutations and methodologies used for CF testing, many labs offer a CF testing panel that is applied to both carrier screening and diagnostic cases. One controversial component of the recommended panel is I148T, which was initially classified as a severe CF mutation. However, in vitro studies indicated that the major CFTR functions are retained by the I148T protein. ${ }^{7}$ Population screening studies revealed that the frequency of I148T is approximately 100 -fold greater in asymptomatic carriers versus CF patients, which is more consistent with a benign variant than a disease-causing mutation. ${ }^{8,9}$ An in-frame deletion in exon 17a, 3199del6, was previously iden-

\footnotetext{
From the ${ }^{I}$ Medical Genetics Laboratories, Department of Molecular and Human Genetics, and the ${ }^{2}$ Department of Pediatrics, Baylor College of Medicine, Houston, Texas.

Benjamin B. Roa, PhD, Medical Genetics Laboratories, Baylor College of Medicine, One Baylor Plaza, NAB 2015, Houston, TX 77030.

Received: March 15, 2004.

Accepted: June 1, 2004.
}

DOI: 10.1097/01.GIM.0000139508.61701.BD tified in association with I148T; moreover, I148T was found to be associated with a severe CF phenotype only when present in a complex haplotype of I148T/3199del6/9T, paired with a severe CF mutation on the opposite chromosome. . $^{4,8}$ The clinical significance of the 3199del6/I148 haplotype is unclear from the limited data available, because the few laboratories that test for 3199del6 mostly do reflex testing limited to patients who are positive for I148T.

Our laboratory developed and implemented an expanded CF panel using the MALDI-TOF mass spectrometry platform (Sequenom). Our current panel of $51 \mathrm{CF}$ mutations contains the 25 core mutations ${ }^{6,10}$ plus 26 additional mutations including 3199del6. This MALDI-TOF assay offers high-throughput testing of patient samples for multiple alleles with high sensitivity and accuracy. Moreover, our testing strategy evaluates each patient's 3199del6 status independently of I148T, which is useful for refining genotype-phenotype correlations. We report on the unusual molecular findings of a 3-year-old patient with a clinical diagnosis of CF. The implications of these findings for CF molecular testing practices are discussed.

\section{MATERIALS AND METHODS}

\section{Patient samples}

Since implementation of our MALDI-TOF mass spectrometry protocol, 5368 individual samples were submitted to our laboratory for $\mathrm{CF}$ testing. The stated indications for testing are listed in Table 1. The compound heterozygous patient described in this study was submitted for diagnostic testing, and parental blood samples were obtained to facilitate interpretation. An additional 11 patients who were previously identified as I148T carriers by allele-specific oligonucleotide (ASO) anal- 
Table 1

Summary of 5368 consecutive cases tested by MALDI-TOF Mass Spectrometry

\begin{tabular}{rlccc}
\hline No. & Indication for testing & No. positives (Freq) & I148T & 3199del6 \\
\hline 4839 & Carrier screening & $173(3.5 \% \approx 1 / 28)$ & 16 & 1 \\
155 & Diagnostic & $30(19.4 \% \approx 1 / 5)$ & 0 & 1 \\
34 & Parental/FEB ${ }^{a}$ & $3(8.8 \%)$ & 1 & 0 \\
10 & Prenatal study & $5(50 \%)^{b}$ & 0 & 0 \\
53 & Family history & $20(37.7 \%)$ & 0 & 0 \\
277 & Not specified & $10(3.6 \%)$ & 0 & 0 \\
\hline
\end{tabular}

${ }^{a}$ Fetal echogenic bowel

${ }^{b}$ Either heterozygous or homozygous positive for parental mutations.

ysis were assessed for 3199del6 status using the expanded MALDI-TOF mass spectrometry panel.

\section{CFTR mutation analysis}

Genomic DNA was extracted using the QIAamp 96 DNA Blood Kit. Samples were assayed for a panel of CFTR mutations using the matrix-assisted laser desorption ionizationtime of flight (MALDI-TOF) mass spectrometry platform (Sequenom). This system involves PCR amplification in a 384well format, extension of primers adjacent to the mutation of interest using defined combinations of deoxy- and dideoxynucleotides, and resolution of the corresponding wild-type and mutant primer extension products by their molecular weight. Our current test for 51 CFTR mutations uses 16 multiplexed assays (Table 3), which were designed with the aid of the MassARRAY Assay Design Software (Sequenom). In compliance with ACMG recommendations, this assay provides reflex testing on $\mathrm{R} 117 \mathrm{H}$ positive samples for the $5 \mathrm{~T}, 7 \mathrm{~T}$, and $9 \mathrm{~T}$ polymorphic alleles at the intron 8 polythymidine tract. ${ }^{6}$ Robotic automation was applied at the pre-PCR (Multiprobe II HT, Perkin Elmer and Biomek FX, Beckman) and post-PCR processes (Multimek, Beckman). Reagents and protocols for post-PCR processes were obtained from Sequenom. Data col-

Table 2

Description of 28 identified I148T heterozygous individuals ${ }^{a}$

\begin{tabular}{|c|c|c|c|c|c|}
\hline \multirow[b]{2}{*}{ No. } & \multicolumn{3}{|c|}{ Genotype } & \multirow[b]{2}{*}{ Indication } & \multirow[b]{2}{*}{ Ethnicity } \\
\hline & $\mathrm{I} 148 \mathrm{~T}$ & 3199del6 & Others & & \\
\hline 1 & + & - & - & Diagnostic & Not specified \\
\hline 1 & + & - & $\Delta \mathrm{F} 508$ & Parental FEB ${ }^{b}$ & Caucasian \\
\hline 18 & + & - & - & Carrier screen & Caucasian \\
\hline 1 & + & - & - & Carrier screen & Asian Indian \\
\hline 4 & + & - & - & Carrier screen & Not specified \\
\hline 2 & + & - & - & Not specified & Not specified \\
\hline 1 & + & + & - & Carrier screen & Caucasian \\
\hline
\end{tabular}

${ }^{a}$ Includes 17 I148T carriers found in a consecutive dataset by MALDI-TOF Mass Spectrometry (also contributed to ref 14), and 11 I148T carriers previously identified by ASO analysis.

${ }^{b}$ Fetal Echogenic Bowel lection and analysis was facilitated by the MassARRAY RT Software (Sequenom), in combination with our internally developed bridge software to interface the MassARRAY functions with our laboratory information system. This enabled seamless data flow and continuous sample tracking, from patient data entry to reporting of results.

\section{CFTR direct sequencing analysis}

Sequence analysis was performed on our patient and his parents to assess potential unidentified CF mutation(s) that are not included in the 51 mutation panel. Twenty nine PCR primer pairs were designed to amplify the CFTR complete coding region (exons 1-24), plus the promoter region, 2 intronic regions (introns 11 and 19), and the 3 ' untranslated region (3'UTR). PCR primers are located at least 60 bases past the intron/exon boundaries. This sequencing strategy encompasses all mutations described in the current database, ${ }^{4}$ except large deletions and sequences located $>822$ nucleotides upstream of the translation start. PCR products were analyzed on a $2 \%$ agarose gel and purified using the Millipore Multiscreen PCR plate separation system. Bidirectional sequencing was performed using the ABI Big Dye Terminator v.3.1 Cycle Sequencing Kit (Applied Biosystems), and capillary gel electrophoresis was performed using the ABI 3730xl DNA Analyzer (Applied Biosystems). Sequencing results were aligned for comparison to wild type text sequence and chromatograms using the SEQUENCHER 4.1.4 software.

\section{Statistical analysis}

The observed CF carrier frequency in our screening population, and the frequency of I148T in particular, were statistically compared to previously reported CF and I148T carrier frequencies ${ }^{8}$ using the Fisher exact test, with a two-sided $P_{-}$ value reported.

\section{RESULTS}

\section{CFTR mutation panel analysis}

Our expanded CF assay using a MALDI-TOF mass spectrometry system (Sequenom) was initially validated against the ASO hybridization assay that was previously implemented in our laboratory. ${ }^{11}$ A total of 1080 patient samples that were analyzed by ASO were tested in parallel by MALDI-TOF mass spectrometry, and concordant results were obtained with the exception of two cases. One sample was heterozygous for a rare mutation, E60X, which was not included in the smaller (37 mutation) ASO panel. In the second case, an ASO result of a heterozygous G314E mutation was further characterized by MALDI-TOF mass spectrometry as a different allelic variant, G314A (data not shown). DNA sequence analysis confirmed the G314A allele, which is a novel missense variant of inconclusive clinical significance. This finding demonstrates the specificity of the MALDI-TOF assay, which discriminates between oligonucleotide products by their signature molecular 
weights. The MALDI-TOF assay was therefore implemented for routine CF clinical testing in our laboratory.

Since the implementation of our fully integrated MALDITOF mass spectrometry protocol, 5368 patient samples were referred for clinical CF testing. The results of CF testing on this consecutive patient set are listed in Table 1 . Ninety percent of patients referred to our laboratory $(4839 / 5368)$ had carrier screening specified as the indication for testing. Within this group, 173 individuals tested positive for a heterozygous mutation yielding an estimated carrier frequency of approximately 1 in 28 (Table 1 ). As expected, the predominant mutation was $\Delta$ F508 in our dataset. We observed a disproportionately high frequency of the I148T allele among heterozygous carriers with no family history (16/173 CF carriers), compared to patients referred with a definite or possible diagnosis of CF (0/155 CF patients). Our findings are consistent with significantly elevated I148T allele frequencies in asymptomatic carriers versus CF patients by previous reports. ${ }^{8,9}$ In addition, two patients out of 5368 consecutive cases were found to be positive for 3199del6 (Table 1), and details on these two cases are provided in the following sections.

\section{Description of I148T-positive individuals}

A composite group of 28 individuals who tested positive for the I148T allele is listed in Table 2. This group included 17 individuals in our consecutive dataset who were simulta-

\section{Table 3}

Description of the 16 multiplex assays designed to analyze 51 CFTR mutations

\begin{tabular}{cll}
\hline Multiplex & \multicolumn{1}{c}{ Mutations } & \multicolumn{1}{c}{ Exon } \\
\hline 1 & 1078delT, G314E, R352Q, G330X & 7 \\
2 & R347H, R347P, R334W, 1717-1A & 7,11 \\
3 & R553X, S549N, R1162X & 11,19 \\
4 & A559T, R560T, G551D & 11 \\
5 & G542X, S549R, 621+1T, Y122X & 4,11 \\
6 & W1282X, 3876delA, 3905insT, D1152H & 18,20 \\
7 & 3849+4G, 3659delC, 1898+1A & 12,19 \\
8 & 405+1A, 405+3C, 3120A, 3120+1A & 3,16 \\
9 & 394delTT, E60X, G85E & 3 \\
10 & A455E, $\Delta$ F508 ${ }^{a}$ & 9,10 \\
11 & G480C, Q493X, V520F & 10 \\
12 & 711+1T, G178R, 3199del6 & $5,17 \mathrm{a}$ \\
13 & 2143delT, 2184delA, K710X, F316L & 7,13 \\
14 & I148T, R117H, R117C & 4 \\
15 & N1303K, 2789+5A, 3849+10kbT & $14 \mathrm{~b}$, intron19, 21 \\
16 & 4I507 & 10 \\
17 & 5T ${ }^{b}$ & intron 8 \\
\hline
\end{tabular}

$\overline{{ }^{a} \mathrm{~F} 508 \mathrm{C} \text { and I507V, I506V, I506M variants are tested for concurrently with the }}$ $\Delta \mathrm{F} 508$ and $\Delta \mathrm{I} 507$ assays respectively.

${ }^{b} 5 \mathrm{~T}$ reflex testing is performed for R117H- and R117C-positive individuals. neously tested for I148T and 3199del6, plus 11 additional I148T carriers who were previously identified by ASO and subsequently tested by MALDI-TOF. Population screening was the indication for testing among the majority of the I148T carriers (24 out of 28); among these, 23 I148T carriers tested negative for 3199del6. One individual of European Caucasian descent referred to our lab for carrier screening was genotyped positive for both I148T and 3199del6. However, the configuration of the I148T and 3199del6 alleles could not be determined on this asymptomatic individual because parental samples were not submitted for analysis. Of the four remaining I148T carriers who were referred for reasons other than carrier screening, one was a male patient with obstructive azoospermia who was heterozygous positive for I148T and negative for the 3199del6 and 5T alleles. One case involved a clinically healthy female who was tested for CF mutations during pregnancy due to fetal echogenic bowel findings on 2nd trimester ultrasound. She tested heterozygous positive for the I148T and $\Delta$ F508 mutations, and negative for the 3199del6 and the 5T alleles (9T/9T). The cis or trans mutation configuration could not be determined without testing additional family members. These findings are consistent with reports of apparently healthy individuals with a $\Delta$ F508-positive, I148T-positive, and 3199del6-negative genotype. ${ }^{8,9}$ This individual's spouse was also referred for testing and found to be negative for $51 \mathrm{CF}$ mutations and the $5 \mathrm{~T}$ allele $(7 \mathrm{~T} / 7 \mathrm{~T})$.

\section{Identification of a CF patient with a G542X/3199del6 genotype}

In the course of clinical testing with the extended CF panel, we studied a 3-year old Hispanic-American male referred by the Baylor Cystic Fibrosis Care Center at Texas Children's Hospital. This patient was diagnosed with meconium ileus immediately postpartum. A presumptive diagnosis of cystic fibrosis was made, and empiric pancreatic enzyme replacement therapy was begun in the neonatal period. Additional therapies included fat-soluble vitamin supplementation and routine respiratory therapy with bronchodilators and airway clearance. Gibson-Cooke pilocarpine iontophoresis confirmed abnormal sweat chloride concentrations on two separate occasions (90 $\mathrm{mEq} / \mathrm{L}$ and $89 \mathrm{mEq} / \mathrm{L}$ with adequate quantities of sweat collected). Quantitative fecal fat analysis and measurement of fecal elastase were not performed. Chest radiographs taken at three years of age demonstrate mild CF lung disease, although the patient does not exhibit baseline respiratory symptoms at this age. The patient's mother reported that his stools were unchanged if enzyme doses were missed, and that she had discontinued therapy without consulting with the medical team. He has grown well at greater than the 95th percentile for both height and weight, reportedly produces normal stools despite lack of pancreatic enzyme replacement, and therefore appears to be pancreatic sufficient.

Molecular testing by MALDI-TOF mass spectrometry identified one copy of the 3199del6 allele in this patient, as shown in Figure 1 . This assay also demonstrated heterozygosity for the G542X mutation, and reflex testing for the $5 \mathrm{~T}$ variant at CFTR intron 8 showed a genotype of 7T/9T in this patient (data not 


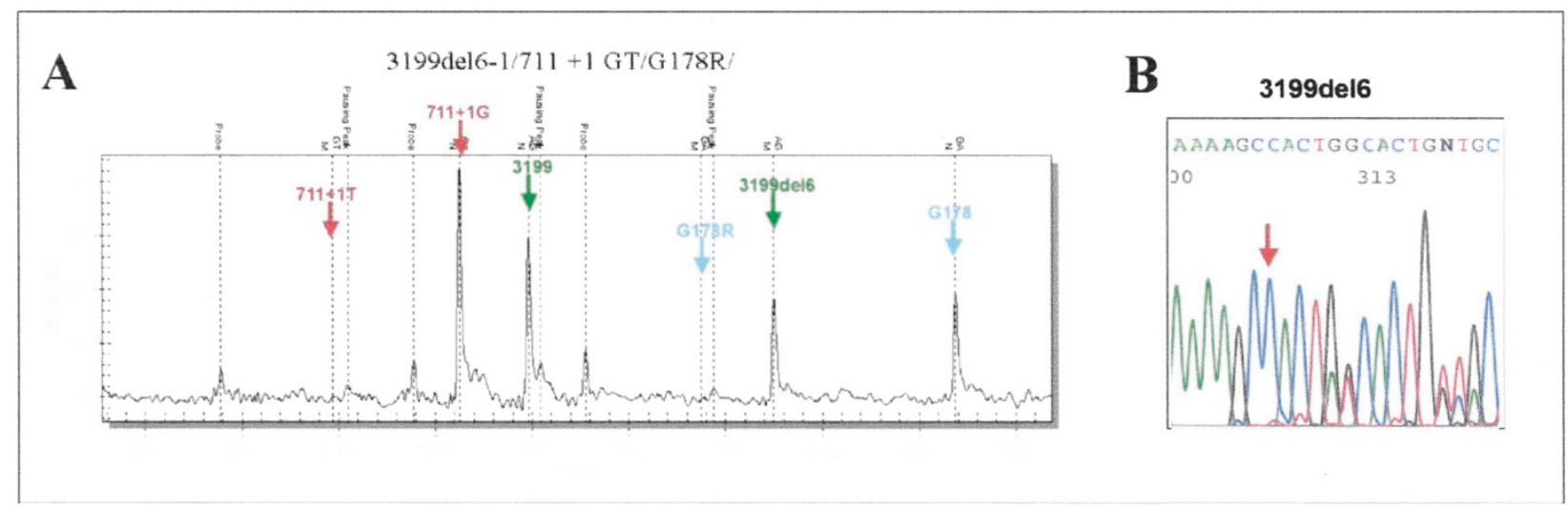

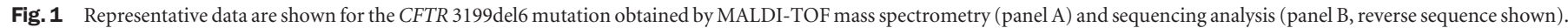
The mass spectrometry chromatogram indicates the expected wild-type and mutant peaks for each mutation in the multiplex CF assay shown ( 31999 del6, G178R, $711+1 \mathrm{~T})$.

shown). Compound heterozygosity for the 3199del6 and G542X mutations was confirmed by parental studies, which identified 3199del6 in the patient's mother and G542X in the father. Both parents and the child shared a 7T/9T genotype, which precluded setting phase for these alleles.

To address the possibility of another unidentified mutation in our patient, full sequence analysis of the CFTR gene was performed. This consisted of bidirectional sequencing of the entire CFTR coding region, the intron 11 and 19 regions that contain known mutations, the promoter region, and the $3^{\prime}$ untranslated region. No definitive mutations were identified in addition to G542X and 3199del6. Sequencing identified twenty three sequence variants, including 15 previously listed polymorphisms. ${ }^{4,12}$ Eight novel sequence variants were also identified at intronic regions, which are likely to represent benign sequence variants (Table 4). Sequencing of regions containing the identified variants in the parental DNA samples confirmed the presence of each sequence variant in at least one parent.

\section{DISCUSSION}

We have successfully implemented an automated MALDITOF mass spectrometry assay for clinical CF testing. This strategy analyzes 51 CFTR mutations concurrently in one tier. The panel includes the 25-mutation ACMG recommended panel plus 26 additional mutations, including 3199del6. Additional mutations were selected on the basis of mutation frequency and technical considerations. Extensive validation studies performed on over 1000 previously tested samples, followed by clinical testing on 5368 consecutive patient samples have demonstrated the system to be extremely accurate, sensitive, and specific. Depending on the sequence context for each mutation, the system can detect novel alleles as aberrant peaks on mass spectrometry chromatograms that can be confirmed by sequencing analysis. Two previously unreported missense alleles were identified: G314A (allelic to G314E) and A455V (allelic to A455E). In addition, the R553G mutation which is allelic to the R553X, was identified in one patient (data not shown). System advantages include high throughput testing for patient samples and mutations, improved turn-aroundtimes, and flexibility in assay design. To complement our enhanced CF mutation panel, we developed sequencing analysis of the CFTR gene as a second-tier test. Sequenced regions include the complete CFTR coding region, the promoter region, two introns known to harbor mutations, and the 3'UTR. This strategy detects mutations involving nucleotide substitutions, small deletions, or insertions; however, sequencing would not rule out large deletions or rearrangements. The combination of these methods provide comprehensive options for CF testing, particularly in patients and families wherein one or both mutations were not identified by mutation panel testing.

The CF mutation frequencies identified in our studies are consistent with previous reports, with the exception of 3199del6. Our mutation detection rate among CF carrier screens was approximately $1 / 28(173 / 4839)$, which is statistically similar to a larger study that differentiated between carrier screening versus diagnostic test results ${ }^{8}(1$ in $24 ; P=0.1$ ). The majority of our carrier screening patients were indicated to be Caucasian, and our overall results are consistent with expectations for demographic uptake and mutation detection rates in a US screening population. Among unaffected individuals who tested positive on carrier screening, the I148T allele was found in approximately $9.2 \%$ of carriers $(16 / 173)$, compared to $\approx 6.4 \%$ from the previously cited study ${ }^{8}(P=0.21)$. In contrast to carrier screening results, none of the 155 diagnostic cases tested by MALDI-TOF mass spectrometry were positive for I148T. This finding is consistent with the significantly elevated I148T frequency in carrier screens versus diagnostic cases, ${ }^{8,9}$ and the relatively small number of diagnostic tests in our study. This observed difference in frequency suggests that I148T is a benign variant, and that 3199 del 6 could instead represent the actual mutation. Up until now, limited data had been available for 3199del6 because systematic analysis was largely based on reflex testing of I148T-positive samples. This prevailing scheme is likely to underestimate the true 3199del6 frequency in both carrier screening and diagnostic cases. In contrast, our assay tests for 3199del6 simultaneously with I148T in an extended panel for CF. 


\section{Buyse et al.}

Table 4

Mutations and sequence variants detected by CFTR sequencing analysis

\begin{tabular}{|c|c|c|}
\hline Nucleotide change & Location & Parental origin \\
\hline \multicolumn{3}{|l|}{ Mutations } \\
\hline G542X & Exon 11 & $\mathrm{~F}$ \\
\hline 3199del6 & Exon $17 \mathrm{a}$ & M \\
\hline polyT status: $7 \mathrm{~T} / 9 \mathrm{~T}$ & Intron 8 & $\mathrm{M}$ and $\mathrm{F}:$ 7T/9T \\
\hline \multicolumn{3}{|l|}{ Known polymorphisms ${ }^{4,12}$} \\
\hline 876-31: 5 TTGA & -31 intron $6 a$ & $\mathrm{M}$ and $\mathrm{F}$ \\
\hline $1001+11 \mathrm{C}>\mathrm{T}$ & +11 intron $6 \mathrm{~b}$ & $\mathrm{M}$ and $\mathrm{F}$ \\
\hline $1525-61 \mathrm{~A}>\mathrm{G}^{a}$ & -61 intron 9 & $\mathrm{M}^{a}$ and $\mathrm{F}^{a}$ \\
\hline $1811+1.6 \mathrm{~kb}+16 \mathrm{~T}>\mathrm{A}^{a}$ & intron 11 & $\mathrm{M}^{a}$ and $\mathrm{F}^{a}$ \\
\hline $1898+152 \mathrm{~T}>\mathrm{A}$ & + 152 intron 12 & $\mathrm{M}$ and $\mathrm{F}$ \\
\hline $\mathrm{T} 854,2694 \mathrm{~T}>\mathrm{G}$ & exon $14 a$ & $\mathrm{M}$ and $\mathrm{F}$ \\
\hline $3041-92 \mathrm{G}>\mathrm{A}$ & -92 intron 15 & M \\
\hline $3121-92 \mathrm{~A} 12 / 13$ & -92 intron 16 & $\mathrm{M}$ and $\mathrm{F}$ \\
\hline $3500-140 \mathrm{~A}>\mathrm{C}$ & -140 intron $17 b$ & M \\
\hline $3601-65 C>A$ & -65 intron 18 & M \\
\hline $4006-200 \mathrm{G}>\mathrm{A}$ & -200 intron 20 & M \\
\hline $4096-283 \mathrm{~T}>\mathrm{C}$ & -283 intron 21 & M \\
\hline $4269-139 \mathrm{G}>\mathrm{A}$ & -139 intron 22 & M \\
\hline $\mathrm{Q} 1463,4521 \mathrm{G}>\mathrm{A}$ & Exon 24 & M \\
\hline $4700 \mathrm{~T} 8 / 9$ & $3^{\prime} \mathrm{UTR}$ & $\mathrm{M}$ and $\mathrm{F}$ \\
\hline \multicolumn{3}{|l|}{ Novel variants } \\
\hline $712-498 \mathrm{~T}>\mathrm{A}^{a}$ & -498 intron 5 & $\mathrm{M}^{a}$ and $\mathrm{F}^{a}$ \\
\hline $712-159 \mathrm{G}>\mathrm{A}$ & -159 intron 5 & $\mathrm{M}$ and $\mathrm{F}$ \\
\hline 875+134_136delTTT & +134 intron $6 a$ & $\mathrm{M}$ and $\mathrm{F}$ \\
\hline $875+641 \mathrm{~A}>\mathrm{G}^{a}$ & +641 intron $6 a$ & $\mathrm{M}^{a}$ and $\mathrm{F}^{a}$ \\
\hline $875+783 \mathrm{~A}>\mathrm{G}^{a}$ & +783 intron $6 \mathrm{a}$ & $\mathrm{M}^{a}$ and $\mathrm{F}^{a}$ \\
\hline $1811+1.6 \mathrm{~kb}-185 \mathrm{~A}>\mathrm{G}^{a}$ & intron 11 & $\mathrm{M}^{a}$ and $\mathrm{F}^{a}$ \\
\hline 2751+86_87insAT & +86 intron $14 \mathrm{a}$ & $\mathrm{M}$ and $\mathrm{F}$ \\
\hline $4374+198 \mathrm{~T}>\mathrm{C}$ & + 198 intron 23 & $\mathrm{M}$ \\
\hline
\end{tabular}

${ }^{a}$ Homozygous sequence variant.

Our findings have significant implications for molecular CF testing. The identification of a CF patient with a compound heterozygous 3199del6/G542X genotype represents the first report of 3199del6 that is not associated with I148T on a CF chromosome. This indicates that 3199del6 is a disease-causing mutation that can occur on a haplotype without I148T. The 3199del6 mutation is therefore an appropriate component of an extended CFTR panel for diagnostic testing. In addition to our Hispanic CF patient, we identified an asymptomatic Caucasian individual who tested positive on carrier screening for 3199del6 and I148T (phase unknown). The observed frequency of 3199del6 in our diagnostic cases (1/155) compared to carrier screens (1/4839) appear consistent with a diseasecausing mutation. Our data underscore the need for larger studies on 3199del6 mutation frequency in different ethnic groups including Hispanics and Caucasians ${ }^{8,13}$ to assess the potential for inclusion in the minimum recommended $\mathrm{CF}$ screening panel. Our results provide additional support to the interpretation of I148T as a benign variant, although its interaction with the 3199del6 mutation is not fully delineated. These findings provide additional support for removing I148T from the ACMG recommended carrier screening panel for $\mathrm{CF}$. For individuals who test positive on cystic fibrosis molecular testing, the recent information needs to be communicated within the context of genetic counseling.

\section{References}

1. Rommens JM, Ianuzzi MC, Kerem B-S, Drumm ML, Melmer G, Dean M et al. Identification of the cystic fibrosis gene: chromosome walking and jumping. Science 1989;245:1059-1065

2. Riordan JR, Rommens JM, Kerem B-S, Alon N, Rozmahel R, Grzelczak Z et al Identification of the cystic fibrosis gene: cloning and characterization of complementary DNA. Science 1989;245:1066-1073.

3. Kerem B-S, Rommens JM, Buchanan JA, Markiewicz D, Cox TK, Chakravarti A et al. Identification of the cystic fibrosis gene: genetic analysis. Science 1989;245:10731080 .

4. University of Washington, Seattle, Washington. GeneTests. Available at: http://www. geneclinics.org/ Accessed 8/6/2004.

5. Hospital for Sick Children, Toronto. Cystic Fibrosis Mutation Database. Available at: http://www.genet.sickkids.on.ca/cftr/ Accessed 8/6/2004.

6. Grody WW, Cutting GR, Klinger KW, Richards CS, Watson MS, Desnick RJ. Laboratory standards and guidelines for population-based cystic fibrosis carrier screening. Genet Med 2001;3:149-154

7. Seibert FS, Jia Y, Mathews CJ, Hanrahan JW, Riordan JR, Loo TW, Clarke DM Disease-associated mutations in cytoplasmic loops 1 and 2 of cystic fibrosis transmembrane conductance regulator impede processing or opening of the channel. Biochemistry 1997;36:111966-11974.

8. Rohlfs EM, Zhou Z, Sugarman EA, Heim RA, Pace RG, Knowles MR et al. The I148T CFTR allele occurs on multiple haplotypes: A complex allele is associated with cystic fibrosis. Genet Med 2002;4:319-323.

9. Strom CM, Huang D, Buller A, Redman J, Crossley B, Anderson B et al. Cystic Fibrosis screening using the College panel: Platform comparison and lessons learned from the first 20,000 samples. Genet Med 2002;4:289-296.

10. Richards CS, Bradley LA, Amos J, Allitto B, Grody WW, Maddalena A et al. Standards and guidelines for CFTR mutation testing. Genet Med 2002;4:379-391.

11. DeMarchi JM, Richards CS, Fenwick RG, Pace R, Beaudet AL. A robotics-assisted procedure for large scale cystic fibrosis mutation analysis. Hum Mutat 1994;4:28190 .

12. Strom CM, Huang D, Chen C, Buller A, Peng M, Quan F et al. Extensive sequencing of the cystic fibrosis transmembrane regulator gene: Assay validation and unexpected benefits of developing a comprehensive test. Genet Med 2003;5:9-14.

13. Wong LJ, Wang J, Zhang YH, Hsu E, Heim RA, Bowman CM et al. Improved detection of CFTR mutations in Southern California Hispanic CF patients. Hum Mutat 2001;18:296-307.

14. Monaghan KG, Highsmith WE, Amos J, Pratt VM, Roa B, Friez M et al. Genotypephenotype correlation and frequency of the 3199del6 cystic fibrosis mutation among I148T carriers: Results from a collaborative study. Genet Med 2004;6:421-425. 